\title{
Oficina de malabares como estratégia para estimular a prática de atividade física e qualidade de vida no Programa Saúde na Escola: relato de experiência
}

\author{
Malabar Workshop as a strategy to encourage the practice of physical activity and quality \\ of life in the School Health Program: experience report \\ Taller Malabar como una estrategia para fomentar la práctica de la actividad física y la \\ calidad de vida en el Programa de Salud Escolar: informe de experiencia
}

Ana Flávia Sela ${ }^{1}$, Amauri Valente Gomes Júnior ${ }^{1}$, Julia do Lago Bataglia ${ }^{1}$, Ana Beatriz Tiemy de Oliveira lamaguti', Marília De Souza Horikawa'1, Vinícius Caporicci Calça', Lourdes Maria Bezerra de Souza Santana ${ }^{1}$, Felipe Thomaz De Aquino1, Isabella Schneider Brito ${ }^{1}$, Marcos Benatti Antunes ${ }^{1 *}$

\section{RESUMO}

Objetivo: Relatar a experiência de intervenção com a oficina de malabares junto aos escolares do $4^{\circ}$ e $5^{\circ}$ ano de uma Escola Municipal de Maringá/PR a fim de estimular a prática de atividade física e melhora na qualidade de vida. Relato de experiência: A intervenção, foi definida por meio de uma oficina de malabares, a partir de uma proposta metodologia lúdica e pedagógica realizada com escolares do $4^{\circ}$ e $5^{\circ}$ ano do ensino fundamental de uma escola municipal de Maringá/PR em conjunto ao Programa Saúde na Escola, a estrutura do trabalho foi organizada em três etapas: a) Indicadores Epidemiológicos; b) Planejamento Participativo com Word Café; e c) Oficina de Malabares. Considerações finais: A experiência proporcionou uma prática lúdica e diferenciada de atividade física, contribuindo para a possibilidade de prevenção de doenças crônicas não transmissíveis e ainda, proporcionou integração entre Unidade Básica de Saúde, Escola Municipal e Instituição de Ensino Superior no Programa Saúde na Escola. A aplicação da Oficina de Malabares apresentou como resultados comportamentais diretos a interação social, fortalecimento da criatividade, trabalho em equipe, além da coordenação motora e equilíbrio dos escolares.

Palavras-chave: Atividade física, Alimentação saudável, Medicina na Arte.

\section{ABSTRACT}

Objective: To report the experience of intervention with the juggling workshop with students from the 4th and 5th year of a Municipal School in Maringá/PR in order to stimulate the practice of physical activity and improve the quality of life. Experience report: The intervention was defined through a juggling workshop, based on a proposal for a playful and pedagogical methodology carried out with 4th and 5th year elementary school students from a municipal school in Maringá/PR together with the Health Program at the School, the work structure was organized in three stages: a) Epidemiological Indicators; b) Participative Planning with Word Café; and c) Malabares workshop. Final considerations: The experience provided a playful and differentiated practice of physical activity, contributing to the possibility of preventing chronic non-communicable diseases and also provided integration between the Basic Health Unit, the Municipal School and the Higher Education Institution in the Health at School Program. The application of the Juggling Workshop showed as direct behavioral results social interaction, strengthening of creativity, teamwork, in addition to motor coordination and balance of students.

Key words: Physical activity, Healthy eating, Medicine in the Arts.

${ }^{1}$ Centro Universitário de Maringá (UNICESUMAR), Maringá - PR. *E-mail: bena77i@gmail.com 


\section{RESUMEN}

Objetivo: Informar sobre la experiencia de intervención en el taller de malabarismo con estudiantes de 4to y 5to año de una Escuela Municipal en Maringá/PR para estimular la práctica de actividad física y mejorar la calidad de vida. Informe de experiencia: La intervención se definió a través de un taller de malabarismo, basado en una propuesta para una metodología lúdica y pedagógica llevada a cabo con estudiantes de primaria de 4to y 5to año de una escuela municipal en Maringá/PR junto con el Programa de Salud en la escuela, la estructura de trabajo se organizó en tres etapas: a) indicadores epidemiológicos; b) Planificación participativa con Word Café; y c) taller de malabares. Consideraciones finales: La experiencia proporcionó una práctica lúdica y diferenciada de actividad física, contribuyendo a la posibilidad de prevenir enfermedades crónicas no transmisibles y también proporcionó integración entre la Unidad Básica de Salud, la Escuela Municipal y la Institución de Educación Superior en el Programa de Salud en la Escuela. La aplicación del Taller de Malabarismo mostró como resultados conductuales directos la interacción social, el fortalecimiento de la creatividad, el trabajo en equipo, además de la coordinación motora y el equilibrio de los estudiantes.

Palabras clave: Actividad Física, Alimentación Saludable, Medicina en las Artes.

\section{INTRODUÇÃO}

No Brasil, as doenças crônicas não transmissíveis (DCNT) contemplam o problema de saúde de maior significância e corresponde a $63 \%$ das mortes no mundo e $72 \%$ no Brasil, atingindo principalmente populações vulneráveis de baixa renda e escolaridade (WHO, 2011; SCHMIDT MI, et al., 2011). As principais classes de DCNT são de origem circulatória, respiratória crônica e ainda comorbidades como câncer e diabetes; essas possuem elementos modificáveis em comum, tais quais tabagismo, álcool, sedentarismo, alimentação não balanceada e obesidade. $O$ aumento da prevalência das doenças crônicas se deve em grande parte às mudanças de hábitos dos brasileiros, os quais predispõem à má alimentação associada ao menor consumo de frutas e verduras frescas e ao maior consumo de industrializados e ao sedentarismo (MINISTÉRIO DA SAÚDE - BRASIL, 2010). A busca pela praticidade e a influência midiática são algumas das responsáveis por essas mudanças (ALMEIDA SS, et al., 2002; VAZ P, et al., 2007).

Durante a fase escolar estendendo-se a adolescência, o contato frequente as mídias bem como as mudanças biopsicossociais e comportamentais nessas fases, há uma tendência em aumento da vulnerabilidade a fatores e comportamentos de risco, comprometendo a qualidade de vida, e ainda com o avanço da idade tendem a ter uma maior predisposição às DCNT (SILVA JEF, et al., 2009).

As consequências dessas mudanças são observadas no aumento da demanda por serviços de saúde à população, com riscos de onerar e inflar esse sistema, sem contar a crescente morbidade dos indivíduos, que além de perderem a qualidade de vida, podem perder a capacidade de trabalho e produtividade, refletindo em toda a economia do país. Por isso, é necessária a prevenção dessas doenças com a abordagem dos fatores de risco modificáveis desde o início da vida perpassando todo o ciclo de vida. Observados esses fatos, o Ministério da Saúde propôs um plano de ação estratégico para o enfrentamento das DCNTs, no qual há propostas de ações intersetoriais, a fim de fortalecer a promoção da saúde aos escolares junto ao Programa Saúde na Escola (PSE), com práticas voltadas para a alimentação saudável, práticas corporais e atividade física, bem como prevenção ao álcool, drogas e tabaco (MINISTÉRIO DA SAÚDE - BRASIL, 2011).

O PSE é uma política intersetorial entre o Ministério da Saúde e o Ministério da educação estabelecido em 2007. Tem por objetivo integrar a educação e a saúde, incrementando a qualidade de vida da população brasileira e visando ao enfrentamento das vulnerabilidades que comprometem o pleno desenvolvimento de crianças e jovens estudantes da rede pública, minimizando situações de risco à saúde de crianças, adolescentes e jovens adultos. As atividades desse programa devem ocorrer no território de abrangência da Estratégia Saúde da Família (ESF) e promover a comunicação entre escolas e unidades de saúde, assegurando a troca de informações sobre as condições de saúde dos estudantes (MINISTÉRIO DA SAÚDE - BRASIL, 2009). 
Pensando nas consequências tanto para o indivíduo como para o sistema de saúde, os acadêmicos de medicina participantes da disciplina de Interação Comunitária II, elaboraram ações que integrassem o PSE e promovessem saúde aos escolares de Maringá/PR. Assim, o objetivo desse estudo relatar a experiência de intervenção com a oficina de malabares junto aos escolares do $4^{\circ}$ e $5^{\circ}$ ano de uma Escola Municipal de Maringá/PR a fim de estimular a prática de atividade física e melhora na qualidade de vida.

\section{RELATO DA EXPERIÊNCIA}

A experiência será descrita em três etapas: a) Indicadores Epidemiológicos; b) Planejamento Participativo; e c) Oficina de Malabares.

\section{Indicadores epidemiológicos}

A experiência proporcionada a partir do plano de ensino da disciplina de Interação Comunitária Il do curso de Medicina do Centro Universitário de Maringá (UNICESUMAR), a qual objetiva o levantamento epidemiológico de uma população abrangida por um mapa delimitado previamente em associação com a equipe de saúde responsável pelas pessoas descritas. A área de estágio das turmas pode ser uma Unidade Básica de Saúde (UBS), escolas públicas, centros educacionais ou ainda outras instituições da área da saúde. No caso descrito, o local foi uma escola de ensino fundamental inserida no território de abrangência de uma UBS do município de Maringá/PR.

A priori foi realizado um levantamento dos indicadores epidemiológicos e, para tal, utilizou-se dois questionários além da mensuração dos dados antropométricos das crianças. O primeiro questionário denominado Marcador de Consumo Alimentar (MCA), disponibilizado pelo Sistema de Vigilância Alimentar e Nutricional (SISVAN), abordava sobre os hábitos de vida e alimentares, como o costume de realizar refeições e utilizar aparelhos eletrônicos concomitantemente, as refeições que costuma fazer ao longo do dia e os alimentos que geralmente ingere. Para analisar a última variável citada, foi perguntado aos alunos o que eles haviam comido no dia anterior, utilizando exemplos listados como feijão, frutas frescas, verduras, legumes, embutidos, bebidas adoçadas, macarrão instantâneo, salgadinhos de pacote, guloseimas e doces (MINISTÉRIO DA SAÚDE - BRASIL, 2011).

Já o segundo questionário dizia respeito à pesquisa de satisfação e insatisfação da imagem corporal dos escolares composto por uma escala de corpos variando de maneira crescente do mais magro para o mais gordo que levava números de 1 a 10. As crianças, então, deveriam apontar para o corpo que considerava parecido com o próprio e posteriormente para o que gostaria de ter.

Durante esse primeiro contato com os escolares, observamos que eles relatavam que durante o final da tarde ou finais de semana, ou seja, períodos que não estavam na escola, a tendência era o consumo de produtos industrializados, incluindo doces e guloseimas. Já no período escolar, as refeições incluíam arroz, feijão, verduras, além de frutas ofertadas todos os dias, outro ponto que chamou bastante atenção é que essa escola municipal oferece quatro refeições diárias (café da manhã, almoço, lanche da tarde e jantar que é servido após as 16:30).

Todos os professores colaboraram para a organização das crianças para passarem nas três estações montadas pelos acadêmicos. Na primeira estação foram aplicados os questionários, na segunda aferido 0 peso e na terceira media a estatura da criança. Na ocasião, observou-se a importância do vínculo e parceria entre escola, UBS e Instituição de Ensino Superior (IES), no que tange a um trabalho focado a saúde das crianças e da comunidade. Após concluir essa etapa, os acadêmicos analisaram os dados e discutiram sobre possíveis intervenções que poderiam ser apresentadas e/ou realizadas na escola para minimizar fatores que contribuem para o desenvolvimento de DCNT. Para isso, organizaram o planejamento participativo.

\section{Planejamento Participativo com Word Café}

O planejamento participativo aconteceu a partir de uma aula com a metodologia chamada "Word café". A referida metodologia objetiva esclarecer e aprimorar, por intermédio de um ambiente como a sala de aula, diálogos entre alunos e professores, envolvendo trocas recíprocas de informações e a efetivação de 
construções de ideias nos diversos campos do conhecimento, sempre de forma coletiva (BROWN J e ISAACS D, 2008). Vale dizer que, para a correta execução da metodologia, é imprescindível a observância de alguns princípios, a saber: a ambiência acolhedora, conexão entre os participantes, escuta, compartilhamento de ideias e os insigths (THE WORLD CAFÉ COMMUNITY, 2015). Nesse contexto, a ambiência foi realizada com um café preparado pelos professores da disciplina Interação Comunitária II, oportunidade em que foi discutido os passos e conceitos do planejamento participativo (Quadro 1).

Quadro 1 - Passos, conceitos e práticas do planejamento participativo na disciplina de Interação Comunitária II do Curso de Medicina do UNICESUMAR, Maringá - PR, 2019.

\begin{tabular}{|c|c|c|}
\hline \multicolumn{3}{|c|}{ PLANEJAMENTO PARTICIPATIVOS } \\
\hline PASSOS & CONCEITO & PRÁTICA \\
\hline Entrada & $\begin{array}{l}\text { Territorialização - reconhecimento } \\
\text { do ambiente e atores do processo }\end{array}$ & $\begin{array}{l}\text { Vinculo formado entre professor, direção da } \\
\text { UBS e direção da escola }\end{array}$ \\
\hline Contratação & $\begin{array}{l}\text { Definição do trabalho coletivo e } \\
\text { reunião das expectativas e da } \\
\text { vontade de mudança }\end{array}$ & $\begin{array}{l}\text { Reunião do professor com os acadêmicos da } \\
\text { disciplina de Interação Comunitária II }\end{array}$ \\
\hline $\begin{array}{l}\text { Preparação/ } \\
\text { Sensibilização }\end{array}$ & Preparação das etapas futuras & $\begin{array}{l}\text { Treinamento orquestrado aos alunos da } \\
\text { Interação Comunitária II para prática de } \\
\text { levantamento e análise de dados. }\end{array}$ \\
\hline $\begin{array}{l}\text { Analise de } \\
\text { cenários }\end{array}$ & $\begin{array}{l}\text { Investigação sobre situação do } \\
\text { sistema - geração e análise de } \\
\text { dados }\end{array}$ & $\begin{array}{l}\text { Levantamento dos indicadores epidemiológicos } \\
\text { e detecção das problemáticas presente entre os } \\
\text { escolares - Pesquisa de hábitos de vida e } \\
\text { alimentares; Pesquisa de satisfação da imagem } \\
\text { corporal. }\end{array}$ \\
\hline $\begin{array}{l}\text { Construção de } \\
\text { cenários }\end{array}$ & $\begin{array}{l}\text { Construção do projeto baseando- } \\
\text { se nos dados gerados }\end{array}$ & $\begin{array}{l}\text { Baseando-se na problemática levantada, foi } \\
\text { instituído a Oficina de Malabares como } \\
\text { intervenção aos escolares. }\end{array}$ \\
\hline $\begin{array}{l}\text { Identificação de } \\
\text { campos de força }\end{array}$ & $\begin{array}{l}\text { Estabelecimento de estratégias de } \\
\text { potencialização dos pontos } \\
\text { positivos e neutralização dos } \\
\text { pontos negativos }\end{array}$ & $\begin{array}{l}\text { Facilitação dinâmica do aprendizado dos } \\
\text { malabares com progressiva adição dos tules } \\
\text { leves e coloridos até etapa final de confecção } \\
\text { das bolinhas feitas de bexiga e painço. }\end{array}$ \\
\hline $\begin{array}{l}\text { Elaboração do } \\
\text { plano }\end{array}$ & $\begin{array}{l}\text { Sistematização dos compromissos } \\
\text { e acordos coletivos }\end{array}$ & $\begin{array}{l}\text { Sistematização da Oficina de Malabares. } \\
\text { Reafirmação de parceria entre grupo de } \\
\text { Interação Comunitária II e Escola Municipal } \\
\text { estabelecendo objetivos, horários, turnos e } \\
\text { cronograma das atividades com o grupo. }\end{array}$ \\
\hline $\begin{array}{l}\text { Acompanhamento } \\
\text { e avaliação }\end{array}$ & $\begin{array}{l}\text { Acompanhamento do processo e } \\
\text { avaliação dos resultados }\end{array}$ & $\begin{array}{l}\text { Realização da Oficina de Malabares com os } \\
\text { escolares. Apresentação das ações realizadas } \\
\text { aos professores e profissionais da UBS e relato } \\
\text { de experiência realizado pelos alunos da } \\
\text { Interação Comunitária II. }\end{array}$ \\
\hline
\end{tabular}

Fonte: Sela AF, et al., 2019. Adaptado de Parente JRF, 2011.

Diante da compreensão dos passos e conceitos do planejamento participativo, os acadêmicos e professor reuniram-se com os professores e coordenadora pedagógica da escola, apresentaram os dados e sugeriram a realização de uma oficina de malabares como intervenção, para estimular a criatividade, coordenação, equilíbrio, lateralidade, concentração, movimento, reflexos e atividade física aos escolares. Quando 
apresentado a atividade, professores e coordenação pedagógica aprovaram a intervenção, demonstrando motivação a atividade apresentada.

\section{Oficina de Malabares}

Após o levantamento dos indicadores e realização do planejamento participativo, a experiência da Oficina de Malabares foi colocada em prática com os escolares do $4^{\circ}$ e $5^{\circ}$ ano. A ação foi realizada durante quatro semanas seguidas na escola, de modo que uma turma por semana participasse. Em cada turma, os alunos foram divididos em grupos de poucos alunos ( 7 a 10) com dois monitores (estudantes de medicina), os quais iriam ensiná-los e acompanhá-los no processo de ensino-aprendizagem.

Primeiramente foi ensinado o malabarismo com os tules (tecidos) leves e coloridos, que demoram mais tempo a cair e auxiliam no treino dos movimentos. Após os alunos terem adquirido habilidade necessária com um tule, adicionava-se progressivamente mais um tule, até completar três. Em seguida, foi iniciada a confecção das bolinhas com saco plástico, balões/bexigas e painço para que as crianças aprendessem e pudessem praticar com as bolinhas, e finalmente atingir o objetivo da oficina.

Durante a intervenção, observou-se que os escolares estiveram bem envolvidos no processo de ensinoaprendizagem, realizaram cada etapa com atenção e esforço de executar os movimentos ensinados pelos acadêmicos e muitos conseguiram chegar no objetivo de lançar os três tules e realizar o malabarismo proposto. Uma das limitações observadas, foi o pouco tempo disponível para execução da oficina, impactando no resultado final de alguns alunos. Em relação a confecção das bolinhas, todos realizaram com ajuda dos acadêmicos. Ao finalizar a oficina de malabares foi confeccionado banners para apresentação aos professores, profissionais da UBS e divulgação em eventos, cumprindo assim, as fases do planejamento participativo, conforme descrito no quadro 1.

\section{DISCUSSÃO}

O projeto no campo da escola permitiu que fossem observadas as condições da Escola Municipal no que se refere à alimentação, implementação de cardápios variados e acolhimento dos alunos. Com base na Política Nacional de Promoção da Saúde (PNPS), publicada pelo Ministério da Saúde em 2006 e redefinida pela Portaria № 2446 em 2014, são temas prioritários da PNPS a formação e educação permanente dos gestores para que possam levar ações de educação em promoção de saúde à população e incluí-la nos espaços de educação permanente; promover alimentação saudável e adequada; práticas corporais e atividades físicas, com a promoção de ações, aconselhamento e incorporação de brincadeiras, jogos, danças populares; enfrentamento do uso do tabaco; enfrentamento do uso abusivo do álcool e outras drogas, entre outros objetivos (MINISTÉRIO DA SAÚDE - BRASIL, 2014).

O Programa Saúde na Escola (PSE) é uma política intersetorial do Ministério da Saúde e do Ministério da Educação, estabelecido em 2007. Os objetivos de tal projeto são planejar, executar e monitorar atividades de prevenção e promoção da saúde. Para cumpri-los, deve-se avaliar as condições de saúde dos educandos, nos âmbitos da saúde nutricional, ocular, bucal, auditiva, clínica e psicossocial.

A importância do PSE é imensa para a vida das crianças e adolescentes, estudantes de escolas públicas, já que o programa age no âmbito de prevenir o abuso de drogas, as DCNT pelo estímulo à atividade física e à alimentação saudável, e também de favorecer o protagonismo desses indivíduos, muitas vezes renegados devido às condições socioeconômicas e autoestima minimizada. Além disso, contribui no acolhimento dos estudantes de modo que diminuam os casos de evasão e abandono à escola (MINISTÉRIO DA SAÚDE BRASIL, 2011).

As práticas circenses chamam a atenção de muitas crianças pelos movimentos, ações e habilidades envolvidas, dentre elas pode-se citar o malabarismo. (AMARANTES AG, 2017). O malabarismo é definido como ato de manipular objetos e manter um ou mais no ar simultaneamente, arremessando-os e recepcionando-os com exatidão e agilidade (LEWBEL A, 2002). Em plena idade de desenvolvimento físico e mental, malabares pode ajudar na construção de coordenação motora, consciência corporal e cognição dos 
pequenos praticantes. Em primeiro lugar, os movimentos que são necessários para a aprendizagem das técnicas requerem a disciplina do treino, a persistência e a consciência corporal, a qual proporciona a compreensão das capacidades do corpo enquanto objeto dinâmico.

O movimento compreende, além da consciência corporal, a estruturação espacial, que permite o reconhecimento do espaço útil para o movimento, a consciência do esforço, a qual prediz a força necessária para a movimentação, e a consciência da relação, que diz respeito a percepção dos objetos e pessoas relacionados ao movimento (GALLAHUE DL e DONNELLY FC, 2008). Sendo assim, a expressão do movimento e a sua consciência são de extrema importância para o bem-estar individual e para 0 conhecimento do próprio corpo no espaço e, muitas vezes, por falta de estímulo renegado a crianças e adolescentes, acarretando na falta de coordenação, equilíbrio e postura inadequada (RENGEL L, 2004). Além disso, a prática da atividade tem importância para o incentivo ao abandono do sedentarismo, uma vez que há movimentos complexos, enquadrando-se como atividade física e, por sua vez, prevenindo as DCNT.

O incentivo à prática de atividades físicas durante a infância e fase pré-puberal tem sua importância na fundamentação teórica de que o exercício realizado próximo ao estirão máximo de crescimento tem efetividade alta para o ganho de massa óssea, sendo que aproximadamente um quarto do osso adulto é formado nos dois anos de velocidade máxima de crescimento. Fatores como esportes e a nutrição associados a adequado consumo de cálcio na dieta são participantes essenciais no processo (ALVES C e LIMA RVB, 2008). Além do incremento da massa óssea e promoção do crescimento físico, outros pontos favoráveis adquiridos são a manutenção positiva no perfil lipídico e o benefício cardiovascular, resultados pertinentes ao debate gerado com o perfil dos escolares e sua direta relação com a DCNTs. Atribuir caráter dinâmico e recreativo à atividade física, como na Oficina de Malabares, torna a sua execução aplicável regularmente por integrar diversão, aprendizado e movimentação corporal, tanto no âmbito escolar quanto fora dela (FERREIRA FC, 2001).

\section{CONSIDERAÇÕES FINAIS}

Conclui-se que a experiência proporcionou uma prática lúdica e diferenciada de atividade física, contribuindo para a prevenção de DCNT e ainda, proporcionou integração entre UBS, escola municipal e IES no PSE. A aplicação da Oficina de Malabares apresentou como resultados comportamentais diretos a interação social, fortalecimento da criatividade, trabalho em equipe, além da coordenação motora e equilíbrio dos escolares. Por meio deste relato de experiência, espera-se futuras produções científicas integrando os cursos da área da ciência da saúde com o PSE.

\section{REFERÊNCIAS}

1. ALVES C, LIMA RVB. Impacto da atividade física e esportes sobre o crescimento e puberdade de crianças e adolescentes. Rev Paul Pediatr 2008; 26(4): 383-91.

2. AMARANTES AG. Malabarismo na Educação Infantil. Matinhos, 2017.

3. ALMEIDA SS, et al. Quantidade e qualidade de produtos alimentícios anunciados na televisão brasileira. Rev. Saúde Pública. 2002; 36(3): 353-355.

4. BRASIL. Ministério da Saúde. Secretaria de Atenção à Saúde. Departamento de Atenção Básica. Saúde na escola / Ministério da Saúde, Secretaria de Atenção à Saúde, Departamento de Atenção Básica. - Brasília: Ministério da Saúde, 2009.

5. BRASIL. Ministério da Saúde. Secretaria de Vigilância em Saúde. Secretaria de Atenção à Saúde. Política Nacional de Promoção da Saúde / Ministério da Saúde, Secretaria de Vigilância em Saúde, Secretaria de Atenção à Saúde. - 3. ed.

- Brasília: Ministério da Saúde, 2010.

6. BRASIL. Ministério da Saúde. Secretaria de Vigilância em Saúde. Departamento de Análise de Situação de Saúde. Plano de ações estratégicas para o enfrentamento das doenças crônicas não transmissíveis (DCNT) no Brasil 2011-2022. Ministério da Saúde. Secretaria de Vigilância em Saúde. Departamento de Análise de Situação de Saúde. - Brasília: Ministério da Saúde, 2011.

7. BRASIL. Ministério da Saúde. Gabinete do Ministro. Portaria nำ2.446, de 11 de novembro de 2014. Redefine a Política Nacional de Promoção da Saúde (PNPS). Diário Oficial da União, Brasília, DF, 2014. 
8. BROWN J, ISAACS D. The World Café: Awakening Collective Intelligence and Committed Action. In M. Torvey Collective Intelligence: Creating a Prosperous World at Peace. Earth Intelligence Network, Virginia. 2008.

9. FERREIRA FC. O malabarismo na escola e sua aplicabilidade prática. Curitiba, 2001.

10. GALLAHUE DL, DONNELLY FC. Educação Física desenvolvimentista para todas as crianças. 4.ed. - São Paulo: Phorte, 2008.

11. LEWBEL, A. Research in Juggling History Research in Juggling History Research in Juggling History original: 2002.

12. PARENTE JRF. Planejamento Participativo em Saúde. SANARE, Sobral, 2011; 10(1): 54-61.

13. RENGEL L. O corpo e possíveis formas de manifestação em movimento. São Paulo: Fundação para o desenvolvimento da educação - Diretorias de projetos especiais, 2004.

14. SCHMIDT MI, et al. Chronic noncommunicable diseases in Brazil: burden and current challenges. Lancet., 2011; 377(9781):1949-61.

15. SILVA JEF, et al. Obesidade e sedentarismo como fatores de risco para doenças cardiovasculares em crianças e adolescentes de escolas públicas de Maringá, PR. Saúde e Pesquisa., 2009; 2(1):41-51.

16. THE WORLD CAFÉ COMMUNITY. Café to go! A quick reference guide for hosting world café. 2015.

17. VAZ P, et al. O fator de risco na mídia. Interface - Comunicação, Saúde, Educação., 2007; 11(21):145-153.

18. WHO - World Health Organization. Global status report on noncommunicable diseases 2010. Geneva: 2011. 